\title{
Utilização da metodologia WebQuest nas aulas de matemática: uma proposta para o estudo de matrizes
}

Camila Faligurski Fim, Pâmela Ceolin Pinto**, Rosana Maria Luvezute Kripka ${ }^{\star \star \star}$

\section{Resumo}

Apresenta-se resultados de uma pesquisa em desenvolvimento, na qual se propõe a utilização do recurso tecnológico digital da WebQuest para o ensino e a aprendizagem de matemática. Também propõe-se a elaboração de uma tarefa para abordagem do tópico matrizes, envolvendo o uso de materiais educacionais, disponibilizados na rede mundial de computadores (WWW), bem como o uso da resolução de problemas, tendo em vista potencializar e facilitar a aprendizagem significativa desse objeto matemático. Pretende-se aplicar a tarefa com estudantes do ensino médio, com o objetivo de avaliar qualitativamente as implicações observadas em termos de aprendizagens ocorridas.

Palavras-chave: Ensino de Matemática. Matrizes. WebQuest.

\section{Introdução}

O ensino e a aprendizagem de matemática têm sido um desafio aos professores. Segundo Barroqueiro e Amaral (2011), essa atividade está se tornando cada vez mais complicada, pois os novos estudantes, que entram para fazer parte do ensino básico ou do ensino superior, nasceram em uma época em que o uso cotidiano das tecnologias digitais é comum, sendo inclusive denominados por "nativos digitais", pertencentes a uma geração denominada por "geração Z". Os autores apontam a necessidade de alternativas para oferecer ambientes agradáveis de aprendizagem em matemática,

Acadêmica do curso de Matemática da UPF. Bolsista de iniciação científica voluntária (Pivic/UPF), Instituto de Ciências Exatas e Geociências, UPF. E-mail: 151179@upf.br

* Acadêmica do curso de Matemática da UPF. Bolsista de iniciação científica voluntária (Pivic/UPF), Instituto de Ciências Exatas e Geociências, UPF. E-mail: 151200@upf.br

*** Mestre em Ciências de Computação e Matemática Computacional pela Universidade de São Paulo, São Carlos, e doutora em Educação em Ciências e Matemática pela Pontifícia Universidade Católica do Rio Grande do Sul. Docente da área de Matemática no Instituto de Ciências Exatas e Geociências da Universidade de Passo Fundo. E-mail: rkripka@upf.br 
que sejam atrativos aos estudantes da geração $\mathrm{Z}$, e destacam o uso de recursos tecnológicos digitais em sala de aula.

A metodologia WebQuest, por suas características, pode contribuir com o processo de ensino e aprendizagem em matemática, pois possibilita explorar diversas abordagens didáticas em educação matemática, tais como: história da matemática, investigação matemática, modelagem matemática, resolução de problemas, etnomatemática em apenas uma atividade (BARROS; NOBRIGA, 2009).

Assim, na presente pesquisa, buscou-se, incialmente, esclarecer aspectos teóricos envolvidos nessa temática, por meio de um mapeamento teórico sobre trabalhos desenvolvidos na área, cujos resultados foram publicados por Fim e Kripka (2017). Nesse sentido, ainda está sendo elaborada uma WebQuest para a abordagem do conceito de matrizes com estudantes do ensino médio, com os objetivos de facilitar sua compreensão e estimular a aprendizagem significativa desse conceito. Portanto, neste texto, apresentam-se os conceitos básicos sobre o recurso e também os materiais selecionados e elaborados para sua aplicação.

\section{Sobre WebQuest}

O recurso tecnológico da WebQuest foi proposto por Bernie Dodge em 1995. Trata-se de uma página na rede mun- dial de computadores (World Wide Web - WWW) que contém informações necessárias e suficientes para que os alunos construam um conhecimento acerca de um assunto escolhido pelo professor.

Segundo Rocha:

Uma WebQuest constitui-se de uma página na $W e b$, desenvolvida pelo professor, que apresenta aos alunos uma determinada tarefa a ser cumprida com base no conteúdo trabalhado em sala de aula. O principal objetivo é aproximar o assunto da realidade do aluno, onde a pesquisa é orientada com base em websites previamente selecionados pelo professor (mas não somente neles) e desenvolvida com base em roteiros elaborados pelo professor tendo como intenção conduzir o aluno ao processo de construção do conhecimento (2007, p. 60).

No modelo de Dodge (1995), podem ser observados ao menos dois tipos de WebQuest: as curtas, que utilizam um pequeno tempo de aplicação, ou seja, demandam em torno de três aulas para se obter o trabalho final, e servem para um conhecimento mais superficial sobre um determinado assunto; e as longas, que se referem ao conhecimento mais específico sobre um tema escolhido, o que demanda um tempo maior para a aplicação, devido aos detalhes que devem ser explorados.

Além disso, uma WebQuest deve ser dividida em etapas a serem cumpridas pelos alunos. Segundo Dio e Andrade (2011), existem seis componentes que a constituem:

- introdução - breve documento que traz informações sobre a atividade; 
- tarefa - descrição dos trabalhos;

- processo - detalhamento de cada atividade a ser realizada;

- recursos/fontes - catálogo das fontes confiáveis para a pesquisa;

- avaliação - maneira como os alunos serão avaliados;

- conclusão - finalização da atividade, sugestão para próximos temas.

O papel do professor nesse tipo de metodologia é extremamente importante. É ele quem elabora, cuidadosamente, todas as etapas da WebQuest, de modo que sejam acessíveis aos estudantes. Também, o professor é o mediador da atividade, é quem estimula o aluno para a prática de pesquisa para a construção do próprio conhecimento, evitando que a aula continue seguindo somente os modelos tradicionais de ensino, em que o professor atua como "transmissor do conhecimento" e os alunos recebem passivamente as informações repassadas.

No mapeamento, realizado por Fim e Kripka (2017), após coleta e investigação qualitativa, verificou-se que sete trabalhos científicos, publicados em periódicos ou em eventos da área, referiam-se a diferentes focos e temas em suas aplicações. Os focos dos artigos variavam entre aplicações diretas do recurso em sala de aula (seis artigos) e em cursos de formação continuada de professores (dois artigos - sendo que um dos trabalhos apresentou os dois focos no decorrer do texto: aplicação em sala de aula e curso de formação continuada de professores). Os temas das aplicações eram matrizes, números reais, geometria espacial, matemática financeira e frações.

Com as aplicações diretas da WebQuest em sala de aula, pode-se observar que, em linhas gerais, essa metodologia de ensino contribuiu para o desenvolvimento de algumas funções cognitivas importantes, características do processo de ensino e aprendizagem, tais como: facilidade na abstração dos conceitos; cooperação e colaboração, propiciadas pelo trabalho em grupo; construção do conhecimento a partir de informações disponibilizadas em rede; entendimento (e não apenas memorização) de fórmulas; entre outras.

Assim, com a investigação teórica inicial sobre os diferentes usos e sobre as potencialidades da WebQuest, foi possível perceber que esse recurso tecnológico digital, quando usado adequadamente, pode favorecer os processos de ensino e aprendizagem em matemática no contexto da sala de aula presencial. Após essas constatações, surgiu a ideia de elaboração de uma tarefa que envolvesse o uso dessa metodologia para analisar o desenvolvimento da atividade e buscar conclusões sobre ela.

\section{Planejamentos e proposições}

Após a leitura dos trabalhos que utilizaram a WebQuest como metodologia de ensino em suas atividades, o grupo de 
pesquisa resolveu elaborar uma tarefa que envolvesse o uso dessa tecnologia a fim de promover o contato de alunos do segundo ano do ensino médio de uma escola de Passo Fundo, RS, com esse recurso. Destaca-se que o mapeamento realizado contribuiu significativamente tanto para a coleta de ideias quanto para a aprendizagem sobre o recurso, suas principais características e etapas.

Na elaboração da WebQuest, o primeiro passo foi verificar como era realizado o processo de construção de uma página da web, buscando, inclusive, verificar quais as dificuldades encontradas na elaboração e na inserção das tarefas na página. Dentre as opções existentes, escolheu-se trabalhar com a construção de páginas na plataforma Google Sites (2010), a qual disponibiliza um aplicativo para construção automática de uma WebQuest, que permite ao usuário a criação gratuita da estrutura básica na qual são inseridos os materiais ou hiperlinks que devem orientar as pesquisas dos estudantes.

Além disso, também foi necessário escolher um conteúdo para ser abordado via WebQuest, visto que esse é um recurso tecnológico que possibilita a construção do conhecimento. Após algumas discussões sobre temas de interesse, o grupo selecionou o estudo de matrizes como objeto de ensino. Salienta-se que um dos motivos que levou a tal escolha foi o fato de o ensino desse tópico ser, ge- ralmente, abordado de modo superficial no ensino médio, visto que os professores restringem-se apenas aos seus aspectos teóricos, desvinculados de usos práticos. Desse modo, a aprendizagem não é significativa, e os estudantes acabam se esquecendo com facilidade dos conceitos. O fato de os estudantes não compreenderem o conceito impede que eles façam usos práticos do conteúdo no cotidiano, tendo em vista a resolução de problemas, como no controle de gastos pessoais, de estoques ou de contabilidade em empresas. Destaca-se que muitos estudantes que ingressam no ensino superior, na área de ciências exatas, geralmente, precisam desse conceito para que possam ampliá-los, ou complexificá-los, em processos de aprendizagem posteriores, em disciplinas específicas, como em álgebra linear.

Após a escolha do tema, foi elaborada a atividade principal da WebQuest, a partir de um vídeo proposto pela Universidade Estadual de Campinas (Unicamp) na forma de Recursos educacionais multimídia para a matemática do ensino médio, no ensino de matrizes. $\mathrm{O}$ vídeo sugerido apresenta um problema intitulado "Cooperativa do leite", ${ }^{1}$ referindo-se a pequenos fazendeiros que conduzem uma cooperativa e pretendem construir um tanque de refrigeração para ser usado coletivamente. Busca-se qual seria a escolha da melhor fazenda para a colocação desse tanque, mas de 
uma maneira otimizada, de modo que a maior viagem de um fazendeiro seja a menor possível. O problema foi resolvido por meio da organização dos dados em uma tabela.

A atividade desenvolvida pelo grupo de pesquisa é baseada no mesmo princípio, porém, retrata a situação de diferentes membros de uma família que pretendem se reunir para uma confraternização anual. Eles são de diferentes cidades e buscam a melhor opção para que as distâncias percorridas no deslocamento sejam as menores. A seguir, apresenta-se a situação-problema criada pelo grupo:

\section{- Problema:}

Todo mês de outubro de cada ano, é tradicional que a família Silva Oliveira faça um grande encontro. Partes dessa família localizam-se nas cidades de Bagé, Caxias do Sul, Erechim, Passo Fundo, Porto Alegre, Santa Maria e Uruguaiana. Neste ano, a família gostaria de escolher a cidade que ficasse mais próxima para todos, ou seja, que todos os membros da família pudessem se deslocar (viajando de carro) o mínimo possível para chegar até o destino final. Com base nos seus conhecimentos sobre o estudo de matrizes e usando recursos da geotecnologia Google Maps ${ }^{\mathrm{TM}}(2017)$, disponibilizada on-line, para calcular a distância entre as cidades, determine qual das cidades citadas seria a melhor escolha, de acordo com as condições desejadas. As cidades estão destacadas no mapa a seguir.

Após a leitura do problema, na página da WebQuest, sugere-se que os estudantes utilizem como exemplo o vídeo citado. Além disso, é dada a seguinte dica: "Organize os dados em uma tabela”. Também, como apoio de orientação, disponibiliza-se o mapa apresentado na Figura 1, esclarecendo que os estudantes podem utilizar o Google Maps ${ }^{\mathrm{TM}}$ (2017) para obter as distâncias mínimas entre as cidades consideradas.

Figura 1 - Localização de cidades do problema proposto

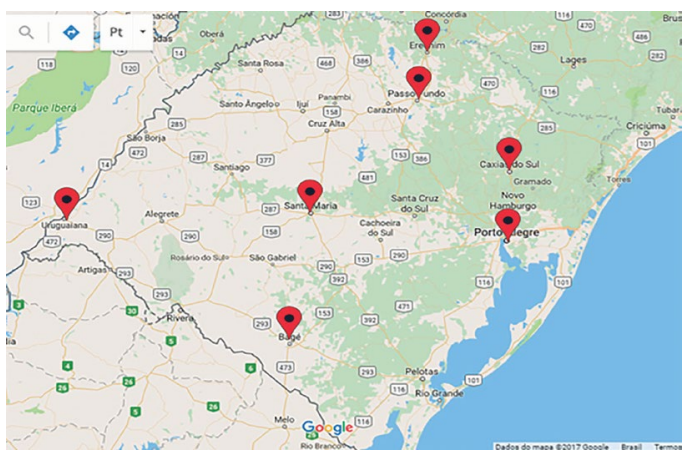

Fonte: Google Maps ${ }^{\mathrm{TM}}(2017)$.

A expectativa é que, a partir da leitura completa do problema, os alunos pensem e organizem uma sequência para a realização das tarefas. $O$ primeiro passo é assistir o vídeo recomendado, pois a atividade é semelhante. Posteriormente, como o problema sugere, os alunos devem usar a geotecnologia Google Maps $^{\mathrm{TM}}$ (2017) para encontrar a distância entre as cidades; em seguida, devem organizar os dados em uma tabela. Na Figura 2 , apresenta-se um exemplo de como encontrar a distância entre uma cidade e outra. 
Figura 2 - Exemplo de obtenção entre distâncias mínimas entre as cidades

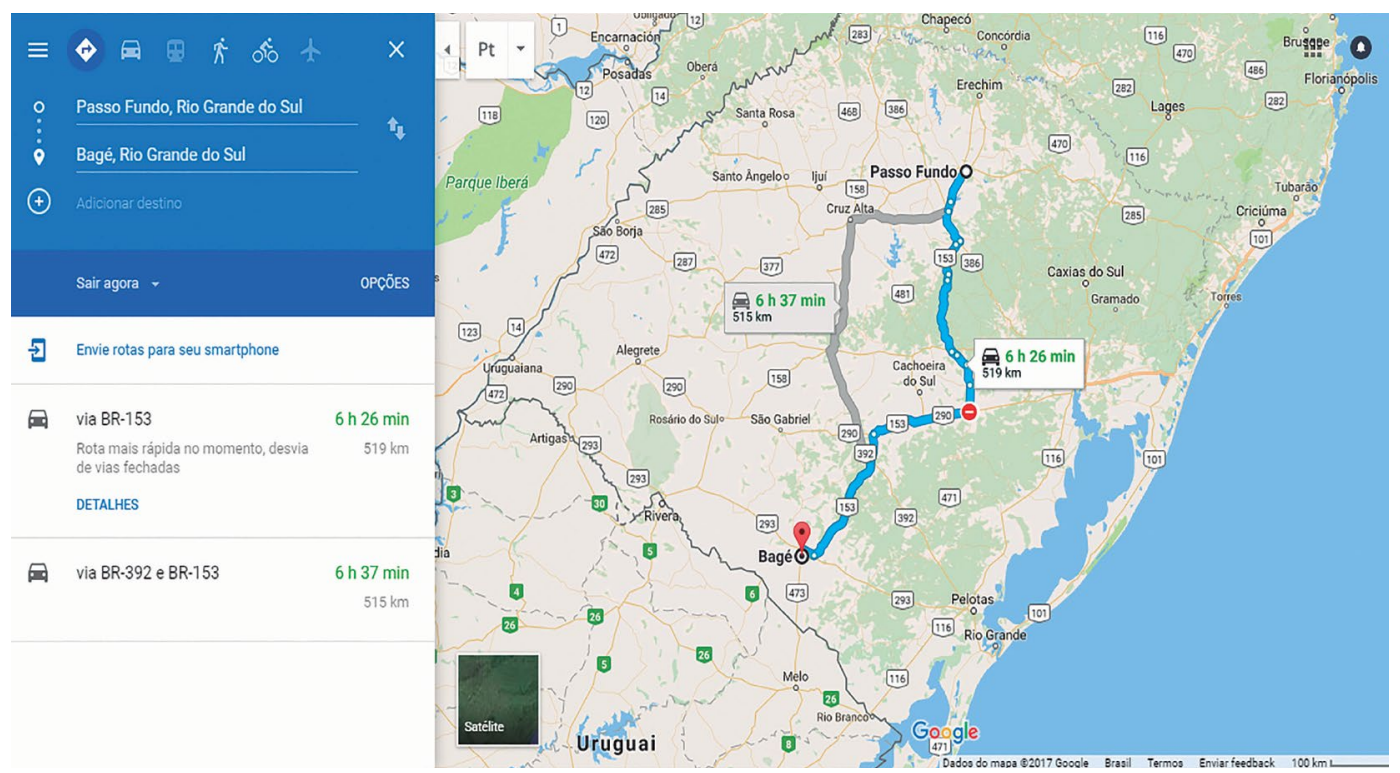

Fonte: Google Maps ${ }^{\mathrm{TM}}$ (2017).

Após encontrarem todas as menores tabela para organizar os dados, como no distâncias possíveis entre uma cidade exemplo apresentado na Tabela 1. e outra, os alunos devem utilizar uma

Tabela 1 - Matriz de distâncias mínimas entre as cidades (em km)

\begin{tabular}{l|r|r|r|r|r|r|r}
\hline \multicolumn{1}{c|}{ X } & Bagé & Caxias & Erechim & Passo Fundo & Porto Alegre & Santa Maria & Uruguaiana \\
\hline Bagé & 0 & 496 & 599 & 518 & 377 & 239 & 388 \\
Caxias & 496 & 0 & 295 & 219 & 127 & 305 & 685 \\
Erechim & 599 & 295 & 0 & 85 & 372 & 356 & 638 \\
Passo Fundo & 518 & 219 & 85 & 0 & 289 & 273 & 559 \\
Porto Alegre & 377 & 127 & 372 & 289 & 0 & 291 & 632 \\
Santa Maria & 239 & 305 & 356 & 273 & 291 & 0 & 382 \\
Uruguaiana & 388 & 685 & 638 & 559 & 632 & 382 & 0 \\
\hline
\end{tabular}

Fonte: elaboração das autoras.

Ao analisar os dados, os alunos devem concluir que, entre as distâncias a serem percorridas, a menor é entre a cidade de Uruguaiana e a cidade de Santa Maria. Portanto, o melhor local para ser realizada a confraternização é na cidade de Santa Maria.

$\mathrm{Na}$ sequência da atividade, o nível de dificuldade aumenta, mas também se assemelha à segunda parte do vídeo 
de orientação. A seguir, apresenta-se a segunda parte da atividade proposta.

$\rightarrow$ Suponha agora que:

- Nove pessoas moram em Bagé;

- Vinte e sete pessoas moram em Caxias do Sul;

- Dezoito pessoas moram em Erechim;

- Trinta pessoas moram em Passo Fundo;

- Trinta e uma pessoas moram em Porto Alegre;

- Doze pessoas moram em Santa Maria;

- Cinco pessoas moram em Uruguaiana.

$\rightarrow$ Buscando otimizar a ida dessas pessoas até 0 encontro, procura-se alocá-las de modo que utilizem o menor número de carros possível, considerando um carro popular de cinco lugares. Responda:
- Quantos carros deverão deslocar-se de cada cidade, considerando a otimização citada acima?

- Com base no problema anterior, determine qual cidade seria a melhor para a realização do encontro anual, considerando agora o número de pessoas que se deslocará até o evento.

Na segunda etapa, os alunos têm de otimizar os participantes, de modo que utilizem o menor número de carros para se deslocar de uma cidade à outra. A primeira pergunta que devem responder serve de auxílio à segunda. Para responder à segunda pergunta, devem utilizar os métodos anteriores e, com base no vídeo de orientação, devem reorganizar os dados em novas tabelas, conforme exemplificado nas Tabelas 2 e 3.

Tabela 2 - Matriz de distâncias mínimas entre as cidades considerando as quantidades de veículos necessários para o transporte de todos (em km)

\begin{tabular}{l|r|r|r|r|r|r|r}
\hline \multicolumn{1}{c|}{ X } & \multicolumn{1}{c|}{ Bagé } & Caxias & Erechim & Passo Fundo & Porto Alegre & Santa Maria & Uruguaiana \\
\hline Bagé & $2 \times 0$ & $6 \times 496$ & $4 \times 599$ & $6 \times 518$ & $7 \times 377$ & $3 \times 239$ & $1 \times 388$ \\
Caxias & $2 \times 496$ & $6 \times 0$ & $4 \times 295$ & $6 \times 219$ & $7 \times 127$ & $3 \times 305$ & $1 \times 685$ \\
Erechim & $2 \times 599$ & $6 \times 295$ & $4 \times 0$ & $6 \times 85$ & $7 \times 372$ & $3 \times 356$ & $1 \times 638$ \\
Passo Fundo & $2 \times 518$ & $6 \times 219$ & $4 \times 85$ & $6 \times 0$ & $7 \times 289$ & $3 \times 273$ & $1 \times 559$ \\
Porto Alegre & $2 \times 377$ & $6 \times 127$ & $4 \times 372$ & $6 \times 289$ & $7 \times 0$ & $3 \times 291$ & $1 \times 632$ \\
Santa Maria & $2 \times 239$ & $6 \times 305$ & $4 \times 356$ & $6 \times 273$ & $7 \times 291$ & $3 \times 0$ & $1 \times 382$ \\
Uruguaiana & $2 \times 388$ & $6 \times 685$ & $4 \times 638$ & $6 \times 559$ & $7 \times 632$ & $3 \times 382$ & $1 \times 0$ \\
\hline
\end{tabular}

Fonte: elaboração das autoras. 
Tabela 3 - Matriz equivalente à apresentada na Tabela 2

\begin{tabular}{l|r|r|r|r|r|r|r}
\hline \multicolumn{1}{c|}{ X } & Bagé & \multicolumn{1}{c|}{ Caxias } & Erechim & Passo Fundo & Porto Alegre & Santa Maria & Uruguaiana \\
\hline Bagé & 0 & 2976 & 2396 & 3108 & 2639 & 717 & 388 \\
Caxias & 992 & 0 & 1180 & 1314 & 889 & 915 & 685 \\
Erechim & 1198 & 1770 & 0 & 510 & 2604 & 1068 & 638 \\
Passo Fundo & 1036 & 1314 & 340 & 0 & 2023 & 819 & 559 \\
Porto Alegre & 754 & 762 & 1488 & 1734 & 0 & 873 & 632 \\
Santa Maria & 478 & 1830 & 1424 & 1638 & 2037 & 0 & 382 \\
Uruguaiana & 776 & 4110 & 2552 & 3354 & 4424 & 1146 & 0 \\
\hline
\end{tabular}

Fonte: elaboração das autoras.

O passo seguinte é a introdução desses materiais no aplicativo para gerar a WebQuest a ser apresentada aos estudantes do ensino médio.

\section{Consideraçōes finais}

Os resultados teóricos parciais indicam que o uso adequado dos recursos digitais disponibilizados pela WebQuest facilita e potencializa a aprendizagem de conceitos matemáticos, pois favorece a ação participativa do estudante na construção do seu conhecimento.

Segundo a percepção das autoras deste trabalho, a utilização de recursos tecnológicos digitais podem contribuir significativamente para o processo de aprendizagem dos estudantes, em diferentes níveis de ensino, pois a criação de novos ambientes de investigação se faz necessária quando o desejo é a proposição de aulas diferenciadas e atraentes.
Participar desse projeto de pesquisa proporcionou aos envolvidos novos conhecimentos sobre possibilidades de uso de recursos tecnológicos digitais em sala de aula. Como essa metodologia era desconhecida pelas integrantes do grupo, destaca-se que o mapeamento realizado foi essencial para dar início à atividade planejada.

Espera-se que a proposta didática, ao ser aplicada no ambiente de ensino escolarizado, corrobore os resultados já apontados em pesquisas anteriores e satisfaça as expectativas, tanto dos docentes quanto dos discentes, relativas à melhoria dos processos de ensino $\mathrm{e}$ aprendizagem de matrizes.

Considera-se importante que novas pesquisas e práticas sejam feitas nesse campo, a fim de confirmar resultados já encontrados e de tentar solucionar ou amenizar os problemas encontrados no processo. 


\section{The use of the WebQuest methodology in maths classes: a proposal for the study of matrices}

\section{Abstract}

The article presents results of a research in development, in which it is proposed to use the digital technological resource of WebQuest for the teaching and learning of mathematics. Also it's proposes the elaboration of a task to approach the topic matrices, which will involve the use of educational materials, made available on the World Wide Web (WWW), as well as the use of problem solving, with a view to enhancing and facilitating learning meaning of this mathematical object. It is intended to apply the task to high school students, with a view to qualitatively assessing the implications observed in terms of learning.

Keywords: Math education. Matrices. WebQuest.

\section{Nota}

1 Disponível em: <http://m3.ime.unicamp.br/recursos/1076>. Acesso em: 12 dez. 2017.

\section{Referências}

BARROS, G. C.; NOBRIGA, J. C. C. WebQuest e educação matemática: um possível caminho à pesquisa escolar. Educação Matemática em Revista, Brasília, DF, v. 27, p. 36-40, ago. 2009.

BARROQUEIRO, C. H.; AMARAL, L. H. O uso das tecnologias da informação e da comunicação no processo de ensino-aprendizagem dos alunos nativos digitais nas aulas de física e matemática. REnCiMa, São Paulo, SP, v. 2, n. 2, p. 123-143, jul./dez. 2011.
DIO, R. G.; ANDRADE, S. V. R. Utilização de WebQuest na aula de matemática. In: PARANÁ. Secretaria de Estado da Educação. Superintendência de Educação. $O$ professor PDE e os desafios da escola pública paranaense, 2007. Curitiba: SEED/PR, 2011. v. 1. (Cadernos PDE). p. 01-31. Disponível em: <http://www. diaadiaeducacao.pr.gov.br/portals/cadernospde/ pdebusca/producoes_pde/2007_unioeste_mat_ artigo_rosana_gagliotti_de_dio.pdf $>$. Acesso em: 29 maio 2017.

DODGE, B. WebQuests: a technique for Internet-based learning. The Distance Educator, San Diego, v. 1, n. 2, p. 10-13, 1995. (Trad. Jarbas Novelino Barato). Disponível em: <http://www. dm.ufscar.br/ jpiton/downloads/artigo_webquest_original_1996_ptbr.pdf $>$. Acesso em: 2 maio 2017.

GOOGLE MAPSTM. Google MapsTM. 2017. Disponível em: <https://www.google.com.br/ maps/preview>. Acesso em: 29 jun. 2017.

FIM, C. F.; KRIPKA, R. M. L. Aprendizagem em matemática por meio de recursos da Webquest: diferentes usos e indicativos. In: CONGRESSO INTERNACIONAL DE ENSINO DA MATEMÁTICA, 7, 2017. Anais... Canoas, RS: Ulbra, 2017. p. 1-13. Disponível em: <http://www. conferencias.ulbra.br/index.php/ciem/vii/paper/ view/6785>. Acesso em: 1 dez. 2017.

GOOGLE SITES. WebQuest Template. 2010. Disponível em: <https://sites.google.com/site/ studentwebquesttemplate/>. Acesso em: $1 \mathrm{dez}$. 2017.

ROCHA, L. R. A. Concepção de pesquisa no cotidiano escolar: possibilidades de utilização da metodologia Webquest na educação pela pesquisa. 2007. 200 f. Dissertação (Mestrado em Educação) - Programa de Pós-Graduação em Educação, Universidade Federal do Paraná, Curitiba, 2007. 